\title{
Modeling of Errors due to Uncertainties in Ultrasound Sensor Locations in Photoacoustic Tomography
}

\author{
Teemu Sahlström, Aki Pulkkinen, Jenni Tick, Jarkko Leskinen and Tanja Tarvainen
}

\begin{abstract}
Photoacoustic tomography is an imaging modality based on the photoacoustic effect caused by the absorption of an externally introduced light pulse. In the inverse problem of photoacoustic tomography, the initial pressure generated through the photoacoustic effect is estimated from a measured photoacoustic time-series utilizing a forward model for ultrasound propagation. Due to the ill-posedness of the inverse problem, errors in the forward model or measurements can result in significant errors in the solution of the inverse problem. In this work, we study modeling of errors caused by uncertainties in ultrasound sensor locations in photoacoustic tomography using a Bayesian framework. The approach is evaluated with simulated and experimental data. The results indicate that the inverse problem of photoacoustic tomography is sensitive even to small uncertainties in sensor locations. Furthermore, these uncertainties can lead to significant errors in the estimates and reduction of the quality of the photoacoustic images. In this work, we show that the errors due to uncertainties in ultrasound sensor locations can be modeled and compensated using Bayesian approximation error modeling.
\end{abstract}

Index Terms-Photoacoustic tomography (PAT), inverse problems, Bayesian methods, error modeling.

\section{INTRODUCTION}

$\mathbf{P}$ HOTOACOUSTIC tomography (PAT) is an imaging modality that utilizes the photoacoustic effect. In PAT, the imaged target is illuminated with a short (typically nanosecond scale) light pulse. Absorption of this light is followed by local thermal expansion and mechanical stress resulting in an initial pressure distribution within the target. This initial pressure relaxes as broadband ultrasound waves that can be measured on the boundary of the target. In the inverse problem of PAT, the initial pressure is estimated from the measured photoacoustic data. For reviews of PAT, its physical principles and applications see e.g. [1], [2].

Various methods for image reconstruction in PAT have been proposed. Some of the most common approaches include

Manuscript received July 18, 2019; revised November 20, 2019; accepted January 6, 2020. This work was supported by the Academy of Finland (projects 314411 and 312342 Centre of Excellence in Inverse Modelling and Imaging), Jane and Aatos Erkko Foundation, and Alfred Kordelin foundation. (Corresponding author: Teemu Sahlström.)

Teemu Sahlström, Aki Pulkkinen, Jenni Tick, and Jarkko Leskinen are with the Department Applied Physics, University of Eastern Finland, 70211 Kuopio, Finland (e-mail: teemu.sahlstrom@uef.fi; aki.pulkkinen@uef.fi; jenni.tick@uef.fi; jarkko.leskinen@uef.fi).

Tanja Tarvainen is with the Department Applied Physics, University of Eastern Finland, 70211 Kuopio, Finland and also with the Department of Computer Science, University College London, WC1E 6BT London, U.K. (e-mail: tanja.tarvainen@uef.fi).

Color versions of one or more of the figures in this paper are available online at http://ieeexplore.ieee.org. series-summation based methods [3], [4], back-projection type approaches [5], [6], time-reversal [7], [8], least-squares and regularized least-squares techniques [9], [10], and a Bayesian approach [11]-[13]. Out of these methods, the seriessummation and back-projection based methods are based on analytic inversion formulas. In practice, these methods are, however, limited to specific geometries such as planar, cylindrical, or spherical measurement geometries. Time-reversal, least-squares, regularized least-squares, and Bayesian methods utilize a numerical solution of the forward problem. These methods are computationally more intensive as the photoacoustic wave-field within the entire domain needs to be computed. They can, on the other hand, be generally applied in arbitrary measurement geometries.

In this work, the inverse problem of PAT is approached in the framework of Bayesian inverse problems [14], [15] following the groundwork described in [11], [13]. In the Bayesian approach to inverse problems, all of the parameters are modeled as random variables. The solution of the inverse problem, i.e. the posterior probability density, is obtained through inference of measurements, forward model, and a prior model for the unknown parameters.

The inverse problem of PAT is ill-posed. The ill-posedness means that even small errors or uncertainties in the measurements or modeling can result in significant errors in the solution of the inverse problem. These errors can appear as artifacts in the reconstructed images. The modeling errors can arise from, for example, the use of a coarse numerical discretization to reduce computational cost. In addition, some assumptions, such as incorrect speed of sound can result in significant errors in the solution of the inverse problem. Furthermore, with experimental setups, errors may originate from uncertainties in the measurement setup geometry. In order to produce accurate photoacoustic images, these modeling errors or uncertainties have to be taken into account. Previously, modeling of errors and their effect in the solution of the inverse problem in PAT have been studied in cases such as variable speed of sound [16]-[18] and finite ultrasound transducer size [19], [20].

Modeling errors due to discretization can often be eliminated by using sufficiently dense discretization, i.e. forward models which can be considered accurate within measurement precision. With practical measurement setups, elimination of the modeling errors can be more challenging. The measurement setup can, for example, be constructed such that the exact modeling of the sensor locations is challenging.

The Bayesian approach to inverse problem facilitates rep- 
resenting and taking into account errors and uncertainties in parameters, models, and measurement geometries [14]. In this work, we investigate errors caused by uncertainties in ultrasound sensor locations in PAT. Further, we study modeling of these errors using Bayesian approximation error modeling. In the approach, modeling errors are approximated as Gaussian and treated as noise in the solution of the inverse problem. The Bayesian approximation error approach has been previously utilized in the optical inverse problem of quantitative PAT in modeling of noise and errors [21], marginalization of scattering [22], and model reduction by using coarse discretization [23]. Furthermore, it has recently been proposed for marginalization of the speed of sound in PAT [18]. In other optical and ultrasonic imaging applications it has been applied, for example, in compensating of errors due to discretization [24], reduction of the physical model [25], domain truncation [26], [27], uncertainties related to geometry and shape [28], [29], and uncertainties in model parameters [30], [31]. Outside biomedical imaging, it has been utilized in other acoustical modeling and inverse problems such as model reduction in aquifer dimension estimation from seismic signals [32], [33]. This paper is one of the first works in which the Bayesian approximation error modeling is utilized in the inverse problem of PAT. Furthermore, this is one of the few studies of Bayesian approximation error modeling where experimental data is used.

The remainder of this paper is organized as follows. The forward problem of PAT is described in Section II. The Bayesian framework and modeling of errors in PAT are described in Section III. Results using simulated and experimental data are presented in Sections IV and V, respectively. Finally, discussion and conclusions are presented in Section VI.

\section{PhOTOACOUSTIC FORWARD MODEL}

Propagation of photoacoustic waves generated by an initial pressure $p_{0}$ in a non-absorbing acoustically homogeneous medium is described by the initial value problem

$$
\left\{\begin{array}{l}
\nabla^{2} p(r, t)-\frac{1}{c^{2}} \frac{\partial^{2} p(r, t)}{\partial t^{2}}=0 \\
p(r, t=0)=p_{0}(r) \\
\frac{\partial}{\partial t} p(r, t=0)=0
\end{array}\right.
$$

where $p(r, t)$ is the pressure, $r$ denotes the spatial position, $t$ is the time, and $c$ is the speed of sound [34]. In practice, the photoacoustic time series, denoted by $p_{t}$, is measured at finite number of time points and positions around the imaged target. In this work, the wave equation is solved with a pseudospectral $k$-space method using the k-Wave MATLAB toolbox [35].

\section{INVERSE PROBLEM}

In PAT, the objective is to estimate the initial pressure $p_{0}$ generated by the photoacoustic effect, when the measured photoacoustic time series $p_{t}$ is given. According to Bayes' theorem, the solution of the inverse problem can be presented as a conditional probability density function of the form

$$
\begin{aligned}
\pi\left(p_{0} \mid p_{t}\right) & =\frac{\pi\left(p_{t} \mid p_{0}\right) \pi\left(p_{0}\right)}{\pi\left(p_{t}\right)} \\
& \propto \pi\left(p_{t} \mid p_{0}\right) \pi\left(p_{0}\right),
\end{aligned}
$$

where $\pi\left(p_{0} \mid p_{t}\right)$ is the posterior probability density, $\pi\left(p_{t} \mid p_{0}\right)$ is the likelihood probability density, $\pi\left(p_{0}\right)$ is the prior probability density, and $\pi\left(p_{t}\right)$ is a normalization constant [14], [15].

\section{A. Conventional error model}

The solution $p$ of the wave equation (1) is linear with respect to the initial pressure $p_{0}$. Utilizing this linear dependency, the observation model of PAT can be written in a discrete form

$$
p_{t}=K p_{0}+e,
$$

where $p_{t} \in \mathbb{R}^{n}$ is a vector of the photoacoustic time series for each sensor, $p_{0} \in \mathbb{R}^{m}$ is a vector describing the initial pressure, and $e \in \mathbb{R}^{n}$ is additive measurement noise. Further, $K \in \mathbb{R}^{n \times m}$ is a discrete forward operator that is assumed to be exact within measurement accuracy. In this work, the forward operator $K$ is constructed by simulating impulse responses for each of the pixels in the reconstructed domain and placing the recorded waveform on the columns of the forward operator $K$ [11]. The impulse responses were simulated using a k-space time-domain method implemented in the k-Wave toolbox. For a matrix-free approach for the inverse problem of PAT, where the forward operator $K$ is not explicitly constructed or stored, see e.g. [13].

Let us now consider the observation model (3). Commonly, the measurement noise is modeled by assuming that the measurement system has some noise level regardless of the imaged target i.e. the noise $e$ and the initial pressure $p_{0}$ are mutually independent. In this case, the likelihood probability distribution can be written as

$$
\pi\left(p_{t} \mid p_{0}\right)=\pi_{e}\left(p_{t}-K p_{0}\right),
$$

where $\pi_{e}(\cdot)$ denotes the probability density of the measurement noise $e$.

Let us model the measurement noise $e$ as Gaussian $e \sim \mathcal{N}\left(\eta_{e}, \Gamma_{e}\right)$, where $\eta_{e}$ is the mean and $\Gamma_{e}$ is the covariance of the measurement noise. The likelihood probability density (4) can then be written as

$$
\pi\left(p_{t} \mid p_{0}\right) \propto \exp \left\{-\frac{1}{2}\left\|L_{e}\left(p_{t}-K p_{0}-\eta_{e}\right)\right\|_{2}^{2}\right\},
$$

where $L_{e}$ is the Cholesky decomposition of the inverse covariance matrix of the measurement noise $\Gamma_{e}^{-1}=L_{e}^{\mathrm{T}} L_{e}$ [14]. The likelihood model (5) is referred to as the conventional error model (CEM).

Let us further model the prior density $\pi\left(p_{0}\right)$ as Gaussian $p_{0} \sim \mathcal{N}\left(\eta_{p_{0}}, \Gamma_{p_{0}}\right)$, where $\eta_{p_{0}}$ is the mean and $\Gamma_{p_{0}}$ is the covariance of the prior distribution. In this case, the posterior probability density takes the form

$$
\begin{aligned}
\pi\left(p_{0} \mid p_{t}\right) \propto \exp \{ & -\frac{1}{2}\left\|L_{e}\left(p_{t}-K p_{0}-\eta_{e}\right)\right\|_{2}^{2} \\
& \left.-\frac{1}{2}\left\|L_{p_{0}}\left(p_{0}-\eta_{p_{0}}\right)\right\|_{2}^{2}\right\},
\end{aligned}
$$


where $L_{p_{0}}$ is the Cholesky decomposition of the inverse covariance matrix of the prior density $\Gamma_{p_{0}}^{-1}=L_{p_{0}}^{\mathrm{T}} L_{p_{0}}$ [14]. Posterior distribution (6) describes a Gaussian distribution [11]

$$
p_{0} \mid p_{t} \sim \mathcal{N}\left(\eta_{p_{0} \mid p_{t}}, \Gamma_{p_{0} \mid p_{t}}\right)
$$

where

$$
\begin{aligned}
\eta_{p_{0} \mid p_{t}} & =\Gamma_{p_{0} \mid p_{t}}\left(K^{\mathrm{T}} \Gamma_{e}^{-1}\left(p_{t}-\eta_{e}\right)+\Gamma_{p_{0}}^{-1} \eta_{p_{0}}\right) \\
\Gamma_{p_{0} \mid p_{t}} & =\left(\Gamma_{p_{0}}^{-1}+K^{\mathrm{T}} \Gamma_{e}^{-1} K\right)^{-1}
\end{aligned}
$$

\section{B. Bayesian approximation error approach}

To produce accurate solutions of the inverse problem, the forward operator $K$ has to model the underlying physics to a sufficient degree of accuracy. In practice, the forward operator can, however, contain modeling errors due to, for example, numerical discretization or uncertainties in the model parameters. In the Bayesian framework, the modeling errors can be taken into account by using the so-called Bayesian approximation error approach [14], [36].

Let us now consider a situation, where the forward operator $K$ is parameterized by some inaccurately known parameter $\varphi$, such as those arising from uncertainties in measurement geometry. The observation model (3) can then be written as

$$
p_{t}=K(\varphi) p_{0}+e .
$$

In practice, however, the random variable $\varphi$ is often fixed to some constant $\varphi \rightarrow \varphi_{0}$ and a computationally approximate forward operator $K\left(\varphi_{0}\right)$ is used instead. Then, the observation model (9) can be written as

$$
\begin{aligned}
p_{t} & =K\left(\varphi_{0}\right) p_{0}+\left(K(\varphi) p_{0}-K\left(\varphi_{0}\right) p_{0}\right)+e \\
& =K\left(\varphi_{0}\right) p_{0}+\varepsilon+e \\
& =K\left(\varphi_{0}\right) p_{0}+n,
\end{aligned}
$$

where $\varepsilon \in \mathbb{R}^{n}$ is the approximation error describing the discrepancy between the accurate and inaccurate models and $n=\varepsilon+e$ is the total error.

Using the observation model (10), the likelihood distribution can be written in an integral form [37]

$$
\pi\left(p_{t} \mid p_{0}\right)=\int \pi_{e}\left(p_{t}-K\left(\varphi_{0}\right) p_{0}-n\right) \pi_{\varepsilon \mid p_{0}}\left(\varepsilon \mid p_{0}\right) \mathrm{d} \varepsilon,
$$

where $\pi_{\varepsilon \mid p_{0}}(\cdot)$ denotes the conditional probability distribution of the approximation error $\varepsilon$ with respect to the initial pressure $p_{0}$. Let us now model the approximation error $\varepsilon$ as Gaussian distributed $\varepsilon \sim \mathcal{N}\left(\eta_{\varepsilon}, \Gamma_{\varepsilon}\right)$, where $\eta_{\varepsilon}$ and $\Gamma_{\varepsilon}$ are the mean and covariance [14]. According to (11), the approximation error $\varepsilon$ is dependent on the initial pressure $p_{0}$. In previous studies it has, however, been shown that ignoring this dependence generally leads to sufficiently accurate models of the approximation error [26], [28], [38]. Thus, we ignore the dependence of $\varepsilon$ on $p_{0}$ i.e. $\varepsilon \mid p_{0} \rightarrow \varepsilon$. The likelihood probability distribution (11) is then a convolution of two Gaussian distributions. Furthermore, the total error $n$ is also Gaussian distributed $n \sim \mathcal{N}\left(\eta_{n}, \Gamma_{n}\right)$ where $\eta_{n}=\eta_{\varepsilon}+\eta_{e}$ and $\Gamma_{n}=\Gamma_{\varepsilon}+\Gamma_{e}$.

Following these assumptions, the likelihood probability density (11) can be written as

$$
\pi\left(p_{t} \mid p_{0}\right) \propto \exp \left\{-\frac{1}{2}\left\|L_{n}\left(p_{t}-K\left(\varphi_{0}\right) p_{0}-\eta_{n}\right)\right\|_{2}^{2}\right\},
$$

where $L_{n}$ is the Cholesky decomposition of the inverse covariance matrix of the total error $\Gamma_{n}^{-1}=L_{n}^{\mathrm{T}} L_{n}$. The error model described by the likelihood density (12) is referred to as the enhanced error model (EEM) [14].

In the case of a Gaussian prior, the posterior density using the EEM can be written as

$$
\begin{aligned}
\pi\left(p_{0} \mid p_{t}\right) \propto \exp \{ & -\frac{1}{2}\left\|L_{n}\left(p_{t}-K\left(\varphi_{0}\right) p_{0}-\eta_{n}\right)\right\|_{2}^{2} \\
& \left.-\frac{1}{2}\left\|L_{p_{0}}\left(p_{0}-\eta_{p_{0}}\right)\right\|_{2}^{2}\right\} .
\end{aligned}
$$

This is a Gaussian distribution, i.e.

$$
p_{0} \mid p_{t} \sim \mathcal{N}\left(\tilde{\eta}_{p_{0} \mid p_{t}}, \tilde{\Gamma}_{p_{0} \mid p_{t}}\right)
$$

where

$$
\begin{aligned}
\tilde{\eta}_{p_{0} \mid p_{t}} & =\tilde{\Gamma}_{p_{0} \mid p_{t}}\left(K\left(\varphi_{0}\right)^{\mathrm{T}} \Gamma_{n}^{-1}\left(p_{t}-\eta_{n}\right)+\Gamma_{p_{0}}^{-1} \eta_{p_{0}}\right) \\
\tilde{\Gamma}_{p_{0} \mid p_{t}} & =\left(\Gamma_{p_{0}}^{-1}+K\left(\varphi_{0}\right)^{\mathrm{T}} \Gamma_{n}^{-1} K\left(\varphi_{0}\right)\right)^{-1} .
\end{aligned}
$$

Approximation error $\varepsilon$ can be determined by, for example, using simulations and a sample based approximation as follows. Let $S=\left\{s^{(1)}, s^{(2)}, \cdots, s^{(N)}\right\}$ be a set of samples drawn from prior distribution of $p_{0}$ and $\varphi^{(l)}$ a sampled value of the uncertain parameter $\varphi$. Mean and covariance of the approximation error $\varepsilon$ can then be computed using the accurate and inaccurate forward models as

$$
\begin{aligned}
& \eta_{\varepsilon}=\frac{1}{N} \sum_{l=1}^{N} \varepsilon^{(l)} \\
& \Gamma_{\varepsilon}=\frac{1}{N-1} \sum_{l=1}^{N} \varepsilon^{(l)}\left(\varepsilon^{(l)}\right)^{\mathrm{T}}-\eta_{\varepsilon} \eta_{\varepsilon}^{\mathrm{T}},
\end{aligned}
$$

where

$$
\varepsilon^{(l)}=K\left(\varphi^{(l)}\right) s^{(l)}-K\left(\varphi_{0}\right) s^{(l)} .
$$

Simulation of the modeling error statistics can be time consuming. However, it needs to be computed only once for a certain measurement geometry and prior distribution.

\section{Simulations}

Effect and compensation of uncertainties in ultrasound sensor locations were studied with simulations using multiple sensor geometries and increasing levels of uncertainty. In the simulations, a circular, $10 \mathrm{~mm}$ diameter region was considered. The speed of sound was set to $c=1500 \mathrm{~m} / \mathrm{s}$. The simulated initial pressure $p_{0}$ contained seven isotropic Gaussian inclusions of various sizes (standard deviations between 400 and $750 \mu \mathrm{m}$, peak amplitude of 1 near the boundary and 0.6 in the middle) illustrated in Fig. 1.

In the simulations, three sensor geometries were studied. The geometries consisted of arcs spanning $360^{\circ}, 180^{\circ}$, and $130^{\circ}$ of the domain boundary with sensor separation of $10^{\circ}$. In this work, these sensor geometries are referred to as $\mathrm{G}_{360}, \mathrm{G}_{180}$, and $\mathrm{G}_{130}$ respectively. The sensors were modeled as ideal point sensors. Sensor positions were defined as cartesian coordinate pairs. In the cases where the coordinates did not match a spatial discretization point, the recorded signals were linearly interpolated using piecewise linear triangular discretization. Visualization of the sensor geometries is presented in Fig. 1. 


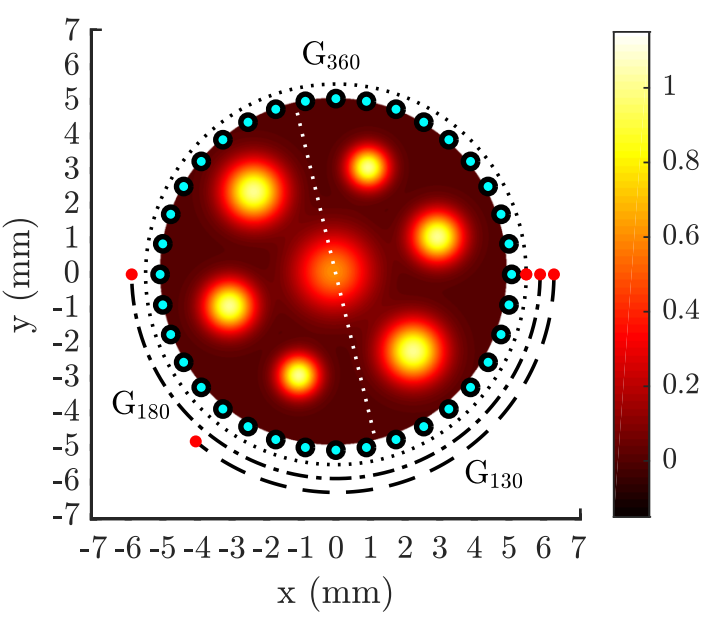

Fig. 1. Simulated initial pressure and sensor geometries. Evenly spaced fullview sensor geometry is indicated as light blue dots. Subsets of these sensors used in geometries $\mathrm{G}_{360}$ (dotted line), $\mathrm{G}_{180}$ (dash-dotted line), and $\mathrm{G}_{130}$ (dashed line) are indicated with the arcs spanning $360^{\circ}, 180^{\circ}$, and $130^{\circ}$ of the target domain. Cross-section used in the visualization of the posterior distribution is shown with a white dotted line.

\section{A. Data simulation}

For data simulation, a square $10.4 \mathrm{~mm} \times 10.4 \mathrm{~mm}$ computational domain was discretized in $832 \times 832$ pixels with a pixel side length of $\Delta x=12.5 \mu \mathrm{m}$. The discretization was extended by an additional $0.425 \mathrm{~mm}$ (34 pixels) thick perfectly matched layer (PML) to absorb reflections on the boundary of the domain. Temporal discretization was chosen according to a Courant-Friedrichs-Lewy (CFL) number 0.3, which corresponded to a time step of $\Delta t=2.5 \mathrm{~ns}$ and sampling frequency of $400 \mathrm{MHz}$. Number of time points was set to $N_{t}=2767$ points resulting in flight time of $6918 \mathrm{~ns}$ and propagation distance of $10.4 \mathrm{~mm}$.

In order to simulate data with uncertainties in sensor locations, the ultrasound sensor locations were perturbed as illustrated in Fig. 2. These altered sensor locations were drawn from generalized uniform distributions of radial and angular values. For the angular case, the altered sensor locations were drawn randomly from three angular distributions $P_{\mathrm{ANG}, 1}$, $\mathrm{P}_{\mathrm{ANG}, 2}$, and $\mathrm{P}_{\mathrm{ANG}, 3}$ shown in Table $\mathrm{I}$. In the radial case, the altered sensor locations were drawn from three distributions $\mathrm{P}_{\mathrm{RAD}, 1}, \mathrm{P}_{\mathrm{RAD}, 2}$, and $\mathrm{P}_{\mathrm{RAD}, 3}$ shown in Table $\mathrm{I}$.

Measurement data was simulated using the wave equation (1) that was numerically solved using the $k$-space timedomain method implemented in the k-Wave toolbox. Gaussian distributed uncorrelated noise with zero mean $\eta_{e}$ and standard deviation $\sigma_{e}$ of $1 \%$ of the maximum simulated peak amplitude was added to the data.

\section{B. Inverse problem}

For the reconstructions, the computational domain was discretized in $135 \times 135$ pixels of side length $\Delta x=78.1 \mu \mathrm{m}$. The computational domain was extended with an additional $1.7 \mathrm{~mm}$ (22 pixels) thick PML-layer. Temporal discretization was chosen according to a CFL-number 0.3, which corresponded to a time step of $\Delta t=15.6 \mathrm{~ns}$ and sampling frequency of $64.0 \mathrm{MHz}$. Number of time points was set to

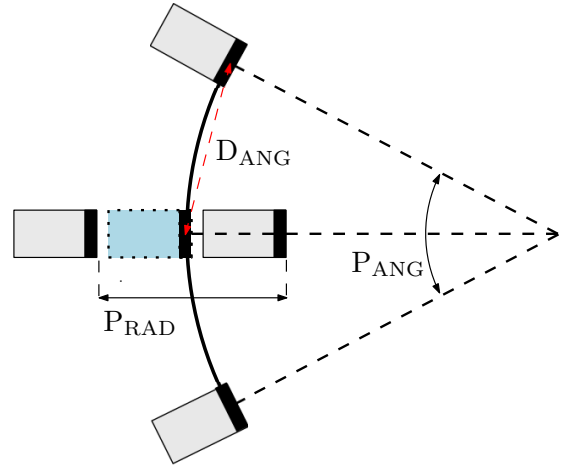

Fig. 2. Visualization of the altered sensor locations in the angular $\left(\mathrm{P}_{\mathrm{ANG}}\right)$ and radial $\left(\mathrm{P}_{\mathrm{RAD}}\right)$ cases. The correct (unaltered) sensor location is indicated as a light blue rectangle with a dotted outline. Cartesian distance $\mathrm{D}_{\mathrm{ANG}}$ between the angularly altered and unaltered sensor location is indicated with the red dotted line.

TABLE I

Angular ( $\mathrm{P}_{\mathrm{ANG}, 1}, \mathrm{P}_{\mathrm{ANG}, 2}$, AND $\left.\mathrm{P}_{\mathrm{ANG}, 3}\right)$ ANd RAdial $\left(\mathrm{P}_{\mathrm{RAD}, 1}, \mathrm{P}_{\mathrm{RAD}, 2}\right.$, And $P_{\text {RAd, } 3}$ ) Distributions Used in Altering of SENSOR Locations

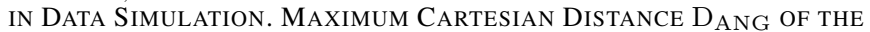
ANGUlar Distributions is SHOWn In SQUARE BRACKETS

\begin{tabular}{lll}
\hline Alteration & \multicolumn{2}{c}{ Distribution } \\
\hline \hline $\mathrm{P}_{\mathrm{ANG}, 1}$ & $\operatorname{Unif}\left(\left[-1^{\circ},-0.5^{\circ}\right] \cup\left[0.5^{\circ}, 1^{\circ}\right]\right)$ & {$[89 \mu \mathrm{m}]$} \\
$\mathrm{P}_{\mathrm{ANG}, 2}$ & $\operatorname{Unif}\left(\left[-2^{\circ},-1^{\circ}\right] \cup\left[1^{\circ}, 2^{\circ}\right]\right)$ & {$[177 \mu \mathrm{m}]$} \\
$\mathrm{P}_{\mathrm{ANG}, 3}$ & $\operatorname{Unif}\left(\left[-3^{\circ},-1.5^{\circ}\right] \cup\left[1.5^{\circ}, 3^{\circ}\right]\right)$ & {$[266 \mu \mathrm{m}]$} \\
$\mathrm{P}_{\mathrm{RAD}, 1}$ & $\operatorname{Unif}([-45.0 \mu \mathrm{m},-22.5 \mu \mathrm{m}] \cup[22.5 \mu \mathrm{m}, 45.0 \mu \mathrm{m}])$ \\
$\mathrm{P}_{\mathrm{RAD}, 2}$ & $\operatorname{Unif}([-89.0 \mu \mathrm{m},-44.5 \mu \mathrm{m}] \cup[44.5 \mu \mathrm{m}, 89.0 \mu \mathrm{m}])$ \\
$\mathrm{P}_{\mathrm{RAD}, 3}$ & $\operatorname{Unif}([-177.0 \mu \mathrm{m},-88.5 \mathrm{~mm}] \cup[88.5 \mu \mathrm{m}, 177.0 \mu \mathrm{m}])$ \\
\hline
\end{tabular}

$N_{t}=437$ resulting in flight time of $6817 \mathrm{~ns}$ and propagation distance of $10.2 \mathrm{~mm}$. For reconstructions, the simulated measurement data was interpolated to the temporal grid used in the reconstructions.

Prior model used in the reconstructions was the Gaussian Ornstein-Uhlenbeck smoothness prior, that is defined by the covariance function

$$
\Gamma_{p_{0}, i j}=\sigma_{p_{0}}^{2} \exp \left\{-\frac{\left\|r_{i}-r_{j}\right\|}{\ell}\right\},
$$

where $\sigma_{p_{0}}$ is the standard deviation, $r_{i, j}$ are locations of pixels, and $\ell$ is the characteristic length [39]. In this work values of $\sigma_{p_{0}}=0.25$ and $\ell=600 \mu \mathrm{m}$ were used. Expected value of the prior density was set to $\eta_{p_{0}}=0.5$.

Measurement error $e$ was modeled as uncorrelated Gaussian distributed with zero mean. The standard deviation of the measurement error was set as $1 \%$ of the maximum positive amplitude of the simulated noiseless data.

In this work, the inverse problem of PAT was solved using three different error models. In the first case, the posterior distribution was solved with the CEM using (7)-(8) and accurately modeled sensor locations, i.e. the accurate model $K(\varphi)$. In the second case, the posterior distribution was solved with the CEM using (7)-(8) and an inaccurate model $K\left(\varphi_{0}\right)$. This corresponded to a situation, where the modeling errors are not taken into account, i.e. $\varepsilon=0$ and $K\left(\varphi_{0}\right) \neq K(\varphi)$. Finally, in the third case, the posterior distribution was solved with the EEM using (14)-(15), an inaccurate forward model $K\left(\varphi_{0}\right)$, 
TABLE II

Relative ERrors $E_{p_{0}}(\%)$ with Respect to the Simulated Initial PRESSURE FOR SENSOR GEOMETRIES $\mathrm{G}_{360}, \mathrm{G}_{180}$, AND $\mathrm{G}_{130}$ AND Angular Uncertainties $\mathrm{P}_{\mathrm{ANG}, 1}, \mathrm{P}_{\mathrm{ANG}, 2}$, AND $\mathrm{P}_{\mathrm{ANG}, 3}$

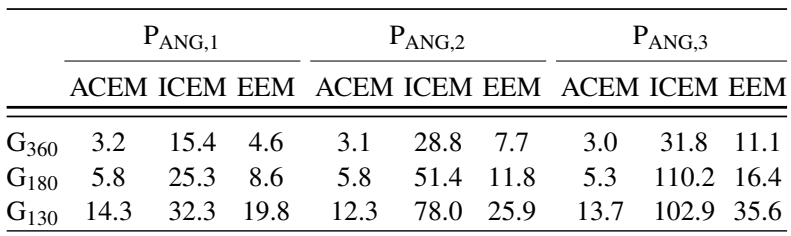

and approximating the modeling errors as Gaussian. In this work, these three approaches are referred to as accurate CEM (ACEM), inaccurate CEM (ICEM), and EEM respectively.

Statistics of the approximation error $\varepsilon$ were computed by simulating forward solutions using the k-Wave toolbox. Estimates for the mean $\eta_{\varepsilon}$ and covariance $\Gamma_{\varepsilon}$ were computed as in (16) $-(18)$ using $N=20000$ samples $s^{(l)}$ drawn from the prior density (19). For these samples, standard deviation, expected value, and characteristic length were set at $\sigma_{p_{0}}=0.25, \eta_{p_{0}}=0.5$, and $\ell=600 \mu \mathrm{m}$ respectively. In the case where the drawn value was negative, which took place only in few occasions, the value was set to zero. Using the samples $s^{(l)}$, forward solutions corresponding to $K\left(\varphi_{0}\right) s^{(l)}$ were computed by using the unaltered sensor geometry. Furthermore, the forward solutions corresponding to $K\left(\varphi^{(l)}\right) s^{(l)}$ were computed using altered sensor locations drawn from uniform distributions between the maximum negative and positive alterations. This meant that, for example, in the case of uncertainty $\mathrm{P}_{\mathrm{ANG}, 1}$, the sensor locations were drawn from a uniform distribution $\mathcal{U}\left(-1^{\circ}, 1^{\circ}\right)$. The sensor locations were drawn independently for every sensor and the locations were drawn repeatedly for each of the samples. Approximation error statistics were computed separately for the cases of angular and radial uncertainties. The number of samples required in teaching the approximation error can be evaluated by inspecting the spectrum of the error model covariance matrix with varying number of samples $N$. Discussion and examples regarding a suitable number of samples $N$ is presented in the Appendix.

Solutions of the inverse problem were visualized by plotting the expected values of the posterior distribution. In addition, expected values on a cross section (Fig. 1) with 99.7\% confidence intervals $( \pm 3$ standard deviations of the posterior distribution) were illustrated. Accuracy of the estimated initial pressure was evaluated by computing relative errors with respect to the simulated initial pressure

$$
E_{p_{0}}=100 \% \frac{\left\|p_{0, \mathrm{SIM}}-p_{0, \mathrm{REC}}\right\|}{\left\|p_{0, \mathrm{SIM}}\right\|},
$$

where $p_{0, \text { SIM }}$ is the simulated (true) initial pressure interpolated to the spatial grid used in the reconstructions and $p_{0, \text { REC }}$ is the expected value of the posterior density, i.e. the estimated initial pressure.

\section{Results - Angular uncertainty}

Expected values of the ACEM-, ICEM-, and EEMreconstructions for the sensor geometries $\mathrm{G}_{360}, \mathrm{G}_{180}$, and $\mathrm{G}_{130}$
TABLE III

Relative ERrors $E_{p_{0}}(\%)$ with Respect to the Simulated Initial PRESSURE FOR SENSOR GEOMETRIES $\mathrm{G}_{360}, \mathrm{G}_{180}$, AND $\mathrm{G}_{130}$ AND Radial Uncertainties $P_{R A D, 1}, P_{R A D, 2}$, AND $P_{R A D, 3}$

\begin{tabular}{|c|c|c|c|c|c|c|c|c|c|}
\hline & & $P_{\text {RAD }, 1}$ & & & $\mathrm{P}_{\mathrm{RAD}, 2}$ & & & RAD,3 & \\
\hline & ACEM & ICEM & EEM & ACEM & I ICEM & EEM & ACEM & ICEM & EEM \\
\hline $\mathrm{G}_{360}$ & 3.0 & 16.9 & 4.9 & 4.1 & 16.4 & 7.5 & 3.4 & 24.1 & 13.5 \\
\hline $\mathrm{G}_{180}$ & 5.7 & 28.8 & 9.8 & 5.5 & 41.1 & 13.6 & 5.4 & 82.4 & 21.7 \\
\hline $\mathrm{G}_{130}$ & 13.5 & 42.8 & 22.5 & 14.1 & 107.9 & 33.1 & 13.5 & 248.8 & 37.4 \\
\hline
\end{tabular}

and angular uncertainties of $\mathrm{P}_{\mathrm{ANG}, 1}, \mathrm{P}_{\mathrm{ANG}, 2}$, and $\mathrm{P}_{\mathrm{ANG}, 3}$ are shown in Fig 3. Corresponding expected values on a cross section (Fig. 1) with $99.7 \%$ credible intervals are shown in Fig. 4. Relative errors $E_{p_{0}}$ of the estimates are presented in Table II.

The results show that the errors caused by the uncertainties in sensor locations grow as a function of increasing level of uncertainty and reducing number of sensors. As it can be seen in Fig. 3, the effect of the uncertainty is smallest in the case of full coverage sensor geometry $\mathrm{G}_{360}$, in which case the inverse problem of PAT is only mildly ill-posed. However, even in this case, the errors are not negligible and distortions can be seen on the boundary of the imaged domain. The errors caused by the uncertainties are considerably larger in the cases of limited-view geometries $\mathrm{G}_{180}$ and $\mathrm{G}_{130}$ as the illposedness of the inverse problem increases. In these cases, the errors due to uncertainties in ultrasound sensor locations can be seen as wave-like artifacts spanning the imaged domain. The credible intervals presented in Fig. 4 show that in the presence of the largest uncertainties, the true values of the initial pressure lie often outside of the primary support of the posterior distribution. The errors caused by uncertainties in sensor locations are, however, well-compensated by Bayesian error modeling. As it can be seen in Fig. 3, the EEM is able to restore the locations and shapes of the true initial pressure even in the case of the largest uncertainty $\mathrm{P}_{\mathrm{ANG}, 3}$. In these situations, the amplitudes of the targets are, however, somewhat reduced in the areas of limited sensor coverage. It should also be noted that, in all of the EEM-reconstructions, the true values lie well within the $99.7 \%$ credible intervals of the posterior distribution.

Calculated relative errors follow a similar trend. That is, the relative errors increase with increasing level of uncertainty and reducing number of sensors. Furthermore, modeling of the errors decreases the relative errors significantly.

\section{Results - Radial uncertainty}

Expected values of the ACEM-, ICEM-, and EEMreconstructions for the sensor geometries $\mathrm{G}_{360}, \mathrm{G}_{180}$, and $\mathrm{G}_{130}$ and radial uncertainties of $\mathrm{P}_{\mathrm{RAD}, 1}, \mathrm{P}_{\mathrm{RAD}, 2}$, and $\mathrm{P}_{\mathrm{RAD}, 3}$ are shown in Fig 5. Corresponding expected values on a cross section (Fig. 1) with $99.7 \%$ credible intervals are shown in Fig. 6. Relative errors $E_{p_{0}}$ of the estimates are presented in Table III.

As it can be seen, the errors caused by radial uncertainties in sensor locations follow a similar trend as in the case of angular 

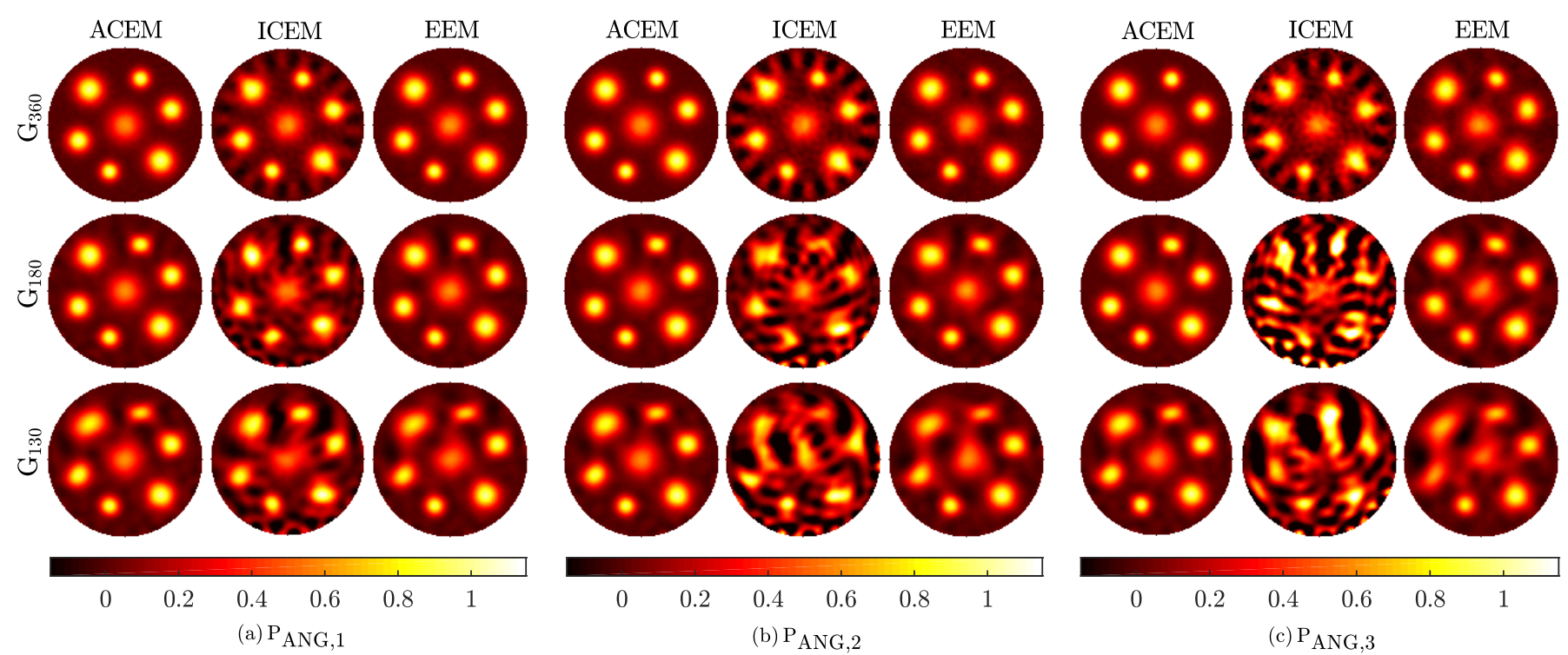

Fig. 3. (a)-(c) Estimated $p_{0}$ for angular uncertainties $\mathrm{P}_{\mathrm{ANG}, 1}-\mathrm{P}_{\mathrm{ANG}, 3}$. For each sub-figure and from left ro right: ACEM, ICEM, EEM, and from top to bottom $\mathrm{G}_{360}, \mathrm{G}_{180}$, and $\mathrm{G}_{130}$.
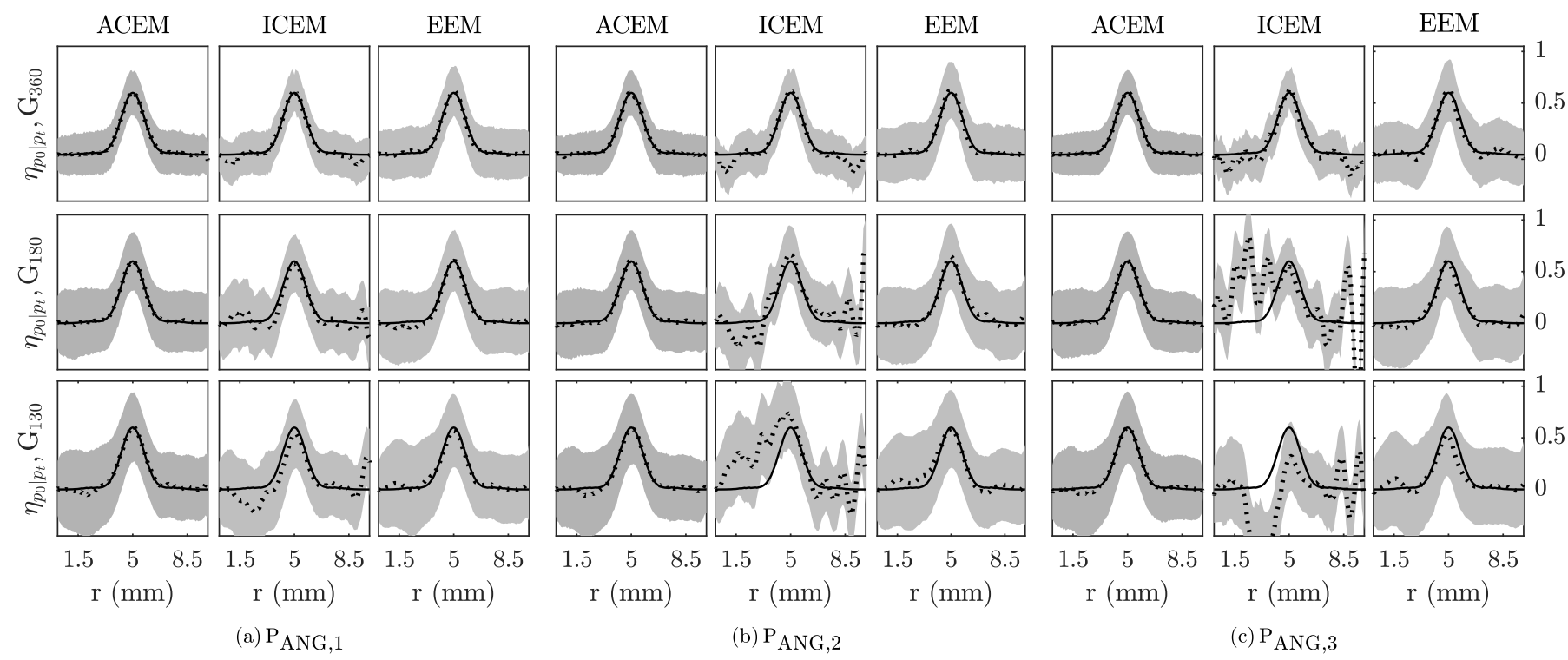

Fig. 4. (a)-(c) Estimated $p_{0}$ (dotted line) and $99.7 \%$ confidence intervals (gray filled area) for angular uncertainties $\mathrm{P}_{\mathrm{ANG}, 1}-\mathrm{P}_{\mathrm{ANG}, 3}$ on a cross section through the domain (from top to bottom on the cross section indicated in Fig. 1). The simulated (true) $p_{0}$ is marked with a solid line. For each sub-figure and from left ro right: ACEM, ICEM, EEM, and from top to bottom $\mathrm{G}_{360}, \mathrm{G}_{180}$, and $\mathrm{G}_{130}$.

uncertainties. The errors, which are effectively compensated by error modeling, grow as a function of increasing uncertainty and decreasing number of sensors. Furthermore, in the cases of CEM and EEM, true values of the initial pressure lie within the primary support of the posterior distribution.

The errors caused by uncertainties in sensor locations are, however, greater than in the case of angular uncertainties. This can be seen when comparing the reconstructions for angular and radial uncertainties of equivalent cartesian distance. For example in the case of uncertainties $\mathrm{P}_{\mathrm{RAD}, 3}$ and $\mathrm{P}_{\mathrm{ANG}, 2}$, the errors caused by radial uncertainty are far greater.

\section{EXPERIMENTAL RESULTS}

The validity of the approach was studied with experimental data measured using a measurement setup described in [40]. The measurement setup comprised of an LED light source (model SST-90-R, Luminus Devices, MA, USA, wavelength $617 \mathrm{~nm}$, pulse energy $5.26 \pm 0.02 \mu \mathrm{J}$, full-width at halfmaximum pulse duration $280 \mathrm{~ns}$ ) and an ultrasound sensor (model V383, Olympus NDT, MA, USA, cylindrically focused, focal distance $33.0 \mathrm{~mm}$, circular aperture diameter 9.53 $\mathrm{mm})$. The sensor was rotated $184^{\circ}$ on an arc of radius $33.8 \mathrm{~mm}$ with $1^{\circ}$ increments. The measured waveforms were averaged over 1024 consecutive pulses. Measurement data was sampled at $50 \mathrm{MHz}$. Schematic of the measurement geometry is shown 



Fig. 5. (a)-(c) Estimated $p_{0}$ for radial uncertainties $\mathrm{P}_{\mathrm{RAD}, 1}-\mathrm{P}_{\mathrm{RAD}, 3}$. For each sub-figure and from left ro right: ACEM, ICEM, EEM, and from top to bottom $\mathrm{G}_{360}, \mathrm{G}_{180}$, and $\mathrm{G}_{130}$
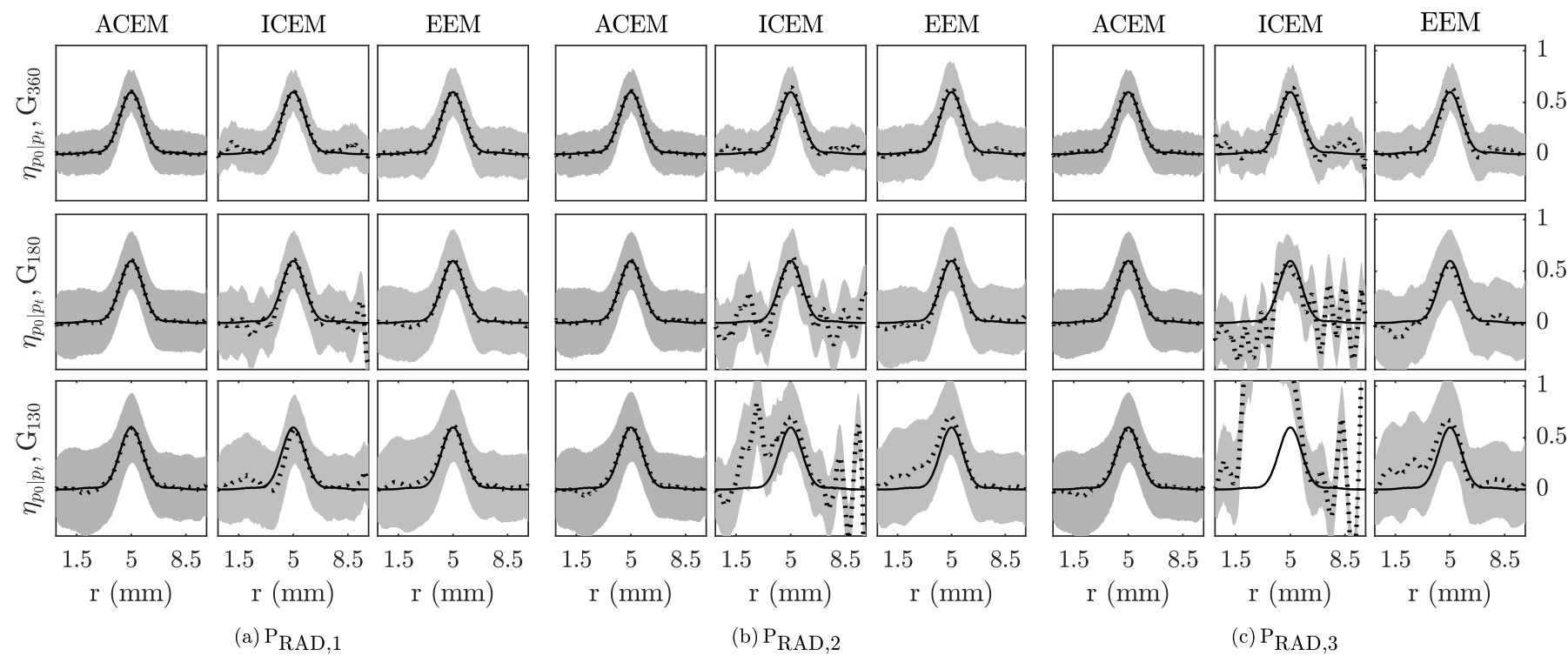

Fig. 6. (a)-(c) Estimated $p_{0}$ (dotted line) and $99.7 \%$ confidence intervals (gray filled area) for angular uncertainties $\mathrm{P}_{\mathrm{RAD}, 1}-\mathrm{P}_{\mathrm{RAD}, 3}$ on a cross section through the domain (from top to bottom on the cross section indicated in Fig. 1). The simulated (true) $p_{0}$ is marked with a solid line. For each sub-figure and from left ro right: ACEM, ICEM, EEM, and from top to bottom $\mathrm{G}_{360}, \mathrm{G}_{180}$, and $\mathrm{G}_{130}$.

in Fig. 7.

Imaged target was constructed from three plastic microcapillary tubes (polyethylene terephthalate glycol; Globe Scientific, NJ, USA) with inner and outer diameters of 0.85 and $1.55 \mathrm{~mm}$ respectively. The tubes were filled with Indian ink (art. no. 44257000, Royal Talens, Apeldoorn, the Netherlands) solution. The tubes were placed side by side (separation of 1 $\mathrm{mm}$ ) on a holder and immersed in water. The target was placed approximately $1.5 \mathrm{~mm}$ towards the measurement arc from the rotational center, such that it was fully inside the coverage of the sensor rotation arc to avoid limited view artifacts [41]. Furthermore, the target was aligned with the z-direction and the sensor was oriented such that the focal line was parallel to the $x-y$ plane. Sinogram and periodogram of the measured data are shown in Fig. 8.

In this work, the reconstructions were computed using three subsets of measurement data sampled from the full dataset. These datasets corresponded to sensor geometries consisting of a $0^{\circ}$ to $180^{\circ}$ sensor arc with detector separations of $6^{\circ}, 8^{\circ}$, and $10^{\circ}$. In this work, these sensor geometries are referred to as $\mathrm{G}_{\mathrm{EXP}, 6}, \mathrm{G}_{\mathrm{EXP}, 8}$, and $\mathrm{G}_{\mathrm{EXP}, 10}$ respectively.

\section{A. Inverse problem}

For the inverse problem, a $72.4 \mathrm{~mm} \times 43.3 \mathrm{~mm} \times 9.8 \mathrm{~mm}$ computational domain was discretized into $384 \times 230 \times 52$ pixels with a pixel side length of $\Delta x=188.4 \mu \mathrm{m}$. 


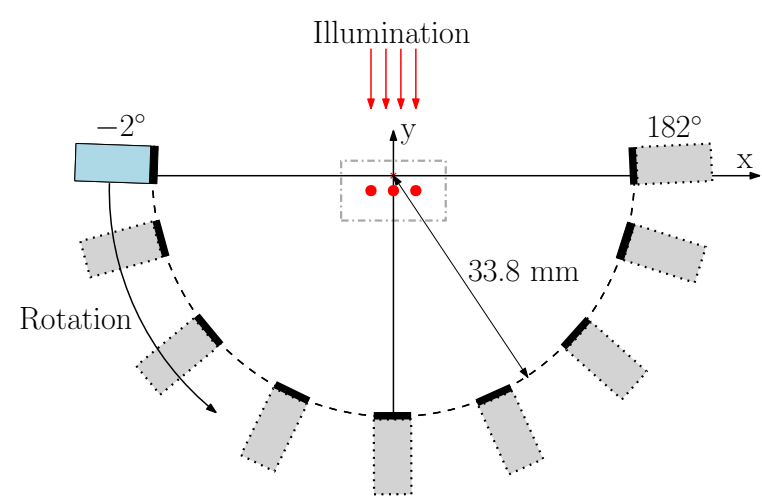

Fig. 7. Schematic of the measurement geometry. Starting position of the ultrasound sensor is shown as the light blue solid rectangle. Partial visualisation of ultrasound sensor rotations are shown as dotted gray rectangles. Reconstructed area containing the imaged target (three red dots) is marked with a gray dash-dotted line.

The spatial discretization was further extended by a $1.5 \mathrm{~mm} \times 2.4 \mathrm{~mm} \times 1.9 \mathrm{~mm}$ thick $(8 \times 13 \times 10$ pixels $)$ PML-layer. Temporal discretization was chosen according to a CFL-number of 0.3 , which corresponded to a time step of $\Delta t=38.2 \mathrm{~ns}$ and sampling frequency of $26.2 \mathrm{MHz}$. Number of time points was set to $N_{t}=897$ resulting in flight time of $34.2 \mu \mathrm{s}$ and propagation distance of $50.8 \mathrm{~mm}$. Speed of sound was determined according to temperature of the water and set to $c=1481.7 \mathrm{~m} / \mathrm{s}$.

The response of the finite-sized sensor was computed by integrating over the ultrasound sensor surface. This was performed by discretizing the surface of the ultrasound sensor into a 124 point equidistant grid with a point separation of $0.8 \mathrm{~mm}$ and averaging the waveforms recorded by each of the points. As in the simulation study, the sensor point locations were defined using cartesian coordinates.

Discrepancy between the model and the data was created by altering the sensor locations radially during the computation of the forward model. The sensor locations were drawn from a discrete uniform distribution of $\mathcal{U}(-188 \mu \mathrm{m}, 0 \mu \mathrm{m}, 188 \mu \mathrm{m})$. Furthermore, the forward operator $K$ was assembled by taking advantage of the z-directional symmetry of the imaged target and assuming that it was homogeneous in the z-direction. This assumption allowed for simulation of $\mathrm{z}$-directional impulse responses i.e. instead of setting one voxel as a source, a $\mathrm{z}$-directional line was used instead. The assumption of $\mathrm{z}$ directional homogeneity allowed for accurate modeling of the concave ultrasound sensor and resulted in a quasi-3D forward model producing 2D images. The advantage of this approach was significantly reduced computational time required for the assembly of the forward operator and the reconstructions. Using the quasi-3D model, the reconstructions were computed in a $7.2 \times 14.9 \mathrm{~mm}(38 \times 79$ pixels $)$ rectangular area (see Fig. 7). The size and location of this area were chosen such that the imaged target was located inside the boundaries of the area.

In the computation of the forward model, the frequency response of the ultrasound sensor was modeled by filtering the simulated signals. The filtering was carried out in the frequency domain using the frequency spectrum provided by
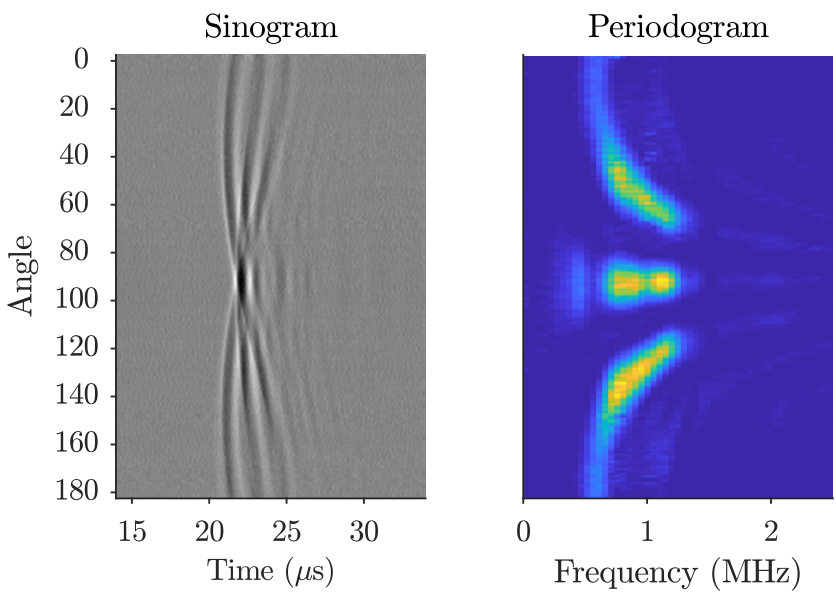

Fig. 8. (left) Sinogram and (right) periodogram of the full measured dataset.

the manufacturer of the ultrasound sensor. The frequency response of the ultrasound sensor was approximately Gaussian with a mean frequency of $3.36 \mathrm{MHz}$ and a full width half maximum of $2.47 \mathrm{MHz}$.

Prior model used in the reconstructions was the OrnsteinUhlenbeck density (19). Standard deviation $\sigma_{p_{0}}$, mean $\eta_{p_{0}}$, and characteristic length $\ell$ of the prior density were set to $\sigma_{p_{0}}=0.04, \eta_{p_{0}}=0.03$, and $\ell=300 \mu \mathrm{m}$ respectively. Mean and standard deviation of the measurement error $e$ were computed from a time window preceding the measured signal. This time frame of $N_{t}=263$ time points consisted entirely of noise. Noise statistics were computed separately for each sensor. Depending on the measurement angle, this resulted in noise levels (standard deviation divided by the maximum recorded amplitude) between $3 \%$ and $22 \%$.

The inverse problem was solved in three cases (ACEM, ICEM, and EEM) described in Section IV. For the ACEMreconstructions, the accurate forward model $K(\varphi)$ was computed using unaltered sensor locations. Further, the inaccurate forward model $K\left(\varphi_{0}\right)$ used in the ICEM- and EEMreconstructions was formed using altered sensor locations.

Statistics of the approximation error $\varepsilon$ were computed by simulating forward solutions similarly as in the simulation study. Estimates for the mean $\eta_{\varepsilon}$ and covariance $\Gamma_{\varepsilon}$ were computed as in (16) - (18) using $N=10000$ 2D-samples drawn from the prior density (19) extended to the z-direction. Standard deviation $\sigma_{p_{0}}$, expected value $\eta_{p_{0}}$, and characteristic length $\ell$ for the sampling were set to $\sigma_{p_{0}}=0.04, \eta_{p_{0}}=0.25$, and $\ell=300 \mu \mathrm{m}$ respectively. For each of the samples, pixel values of less than zero were set to zero. Computing of forward solutions $K\left(\varphi^{(l)}\right) s^{(l)}$ required re-sampling of sensor positions for each of the $N=10000$ samples. In three dimensions, repeated computation of interpolations resulted in a considerable increase in computational time when using the $\mathrm{k}$-Wave toolbox. In order to reduce the computational time, the samples corresponding to $K\left(\varphi^{(l)}\right) s^{(l)}$ were computed as follows. First, the $N$ forward solutions were computed using three distinct simulation geometries $\mathrm{G}_{\mathrm{S} 1}, \mathrm{G}_{\mathrm{S} 2}$, and $\mathrm{G}_{\mathrm{S} 3}$. These three simulation geometries were constructed using constant radial displacements of $-188 \mu \mathrm{m}\left(\mathrm{G}_{\mathrm{S} 1}\right), 0 \mu \mathrm{m}\left(\mathrm{G}_{\mathrm{S} 2}\right)$, and 

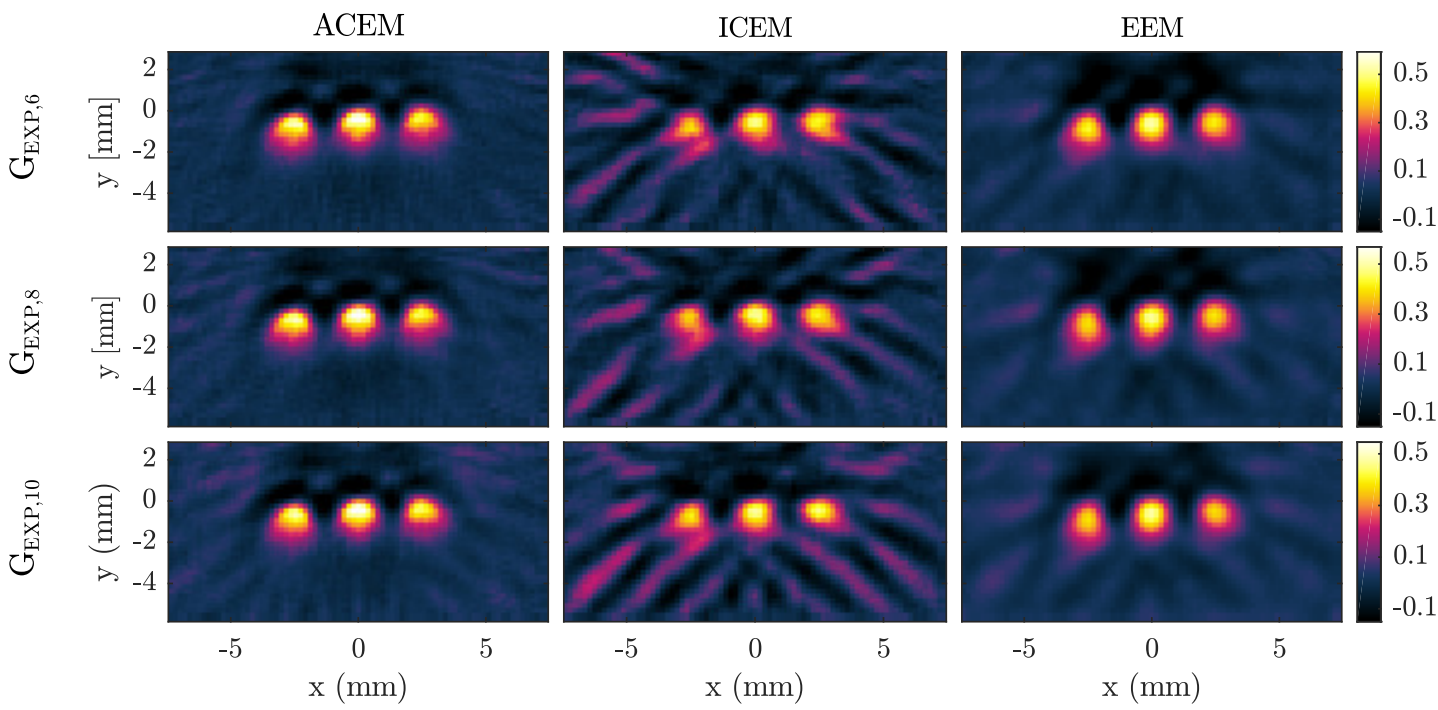

Fig. 9. Estimated $p_{0}$ for sensor geometries $\mathrm{G}_{\mathrm{EXP}, 6}$ (first row), $\mathrm{G}_{\mathrm{EXP}, 8}$ (second row), and $\mathrm{G}_{\mathrm{EXP}, 10}$ (third row) using experimental data. Columns from left to right: ACEM, ICEM, and EEM. Maximum values of the colormaps are scaled row-wise using the maximum values of the ACEM-reconstructions.

$188 \mu \mathrm{m}\left(\mathrm{G}_{\mathrm{S} 3}\right) .188 \mu \mathrm{m}$ corresponds to $43 \%$ of the wavelength of the central frequency of the sensor used in the study. A sample using altered sensor locations was then computed by drawing a radial displacement from a uniform distribution of $\mathcal{U}(-188.0 \mu \mathrm{m}, 188.0 \mu \mathrm{m})$ and interpolating the waveform from the forward solution computed using simulation geometries $\mathrm{G}_{\mathrm{S} 1-\mathrm{S} 3}$. This was done separately for each sensor used in the sensor geometry. Samples $K\left(\varphi_{0}\right) s^{(l)}$ were computed using the three simulation geometries $\mathrm{G}_{\mathrm{S} 1-\mathrm{S} 3}$ and sensor locations used in computation of the forward operator $K\left(\gamma_{0}\right)$.

\section{B. Results}

Expected values of the posterior densities solved using the accurate forward model (ACEM), inaccurate forward model (ICEM), and the inaccurate model with error modeling (EEM) for the sensor geometries $\mathrm{G}_{\mathrm{EXP}, 6}, \mathrm{G}_{\mathrm{EXP}, 8}$, and $\mathrm{G}_{\mathrm{EXP}, 10}$ are shown in Fig. 9. As it can be seen, the effect of the uncertainty in sensor locations results in significant errors in the ICEMreconstructions. The errors can be seen as wave-like artifacts. In addition, the severity of the artifacts increases as the number of sensors decreases.

Regardless of the severity of the artifacts, the effect of the uncertainty in sensor locations is effectively corrected by the EEM. In the EEM-reconstructions, the wave-like artifacts evident in the ICEM-reconstructions are not present and the backgrounds of the images are restored close to the level of ACEM-reconstructions. In addition, the three tubes are clearly visible. Even though the artifacts present in the ICEMreconstructions are effectively compensated for, the EEMreconstructions suffer a from slight reduction of target values and blurring of the tube boundaries.

\section{Discussion AND Conclusions}

In this work, compensation of uncertainties in ultrasound sensor locations in PAT using the Bayesian approximation error modeling was described. The method was studied using both simulated and experimental photoacoustic data. Posterior densities were computed using accurate, inaccurate, and modeling error compensated forward models. The approach was evaluated using various sensor geometries and varying levels of uncertainty.

The results show that modeling errors caused by uncertainties in ultrasound sensor locations can result in significant errors in the solution of the inverse problem. These errors can appear as major artifacts in the reconstructed images. Uncertainties in sensor locations were found to cause significant errors even in the case of smallest studied radial uncertainties of $\pm 45 \mu \mathrm{m}$. The errors were especially evident in the cases of limited-view setups. This is due to the increasing ill-posedness of the inverse problem compared to the full-view setup.

In the simulation studies, uncertainties in radial sensor locations were found to cause larger errors compared to the case of angular uncertainties. Sensitivity to radial uncertainty can be explained by examining the phase shift and wavelength of the impulse responses used in the computation of the forward operator $K$. In the simulation study, the maximum simulated frequency was limited to approximately $10 \mathrm{MHz}$ by the spatial discretization, which corresponded to a wavelength of $150 \mu \mathrm{m}$. Thus, in the case of the smallest radial uncertainty of $\pm 45 \mu \mathrm{m}$, the maximum sensor alteration resulted in a shift of $30 \%$ of the wavelength. This effect was not as prevalent in the case of angular uncertainties. This was due to the fact that initial pressures at or near the center of the measurement geometry produce similar signals regardless of the level of angular uncertainty and are thus less sensitive to uncertainties in sensor locations.

Regardless of the direction of uncertainty, the approximation error approach was found to compensate for the errors caused by the uncertainties. The effects of modeling errors were effectively corrected even in the cases of the most significant uncertainties. In addition, the credibility intervals of the posterior density were wider in the case of error modeling. Furthermore, the expected values of the simulated 


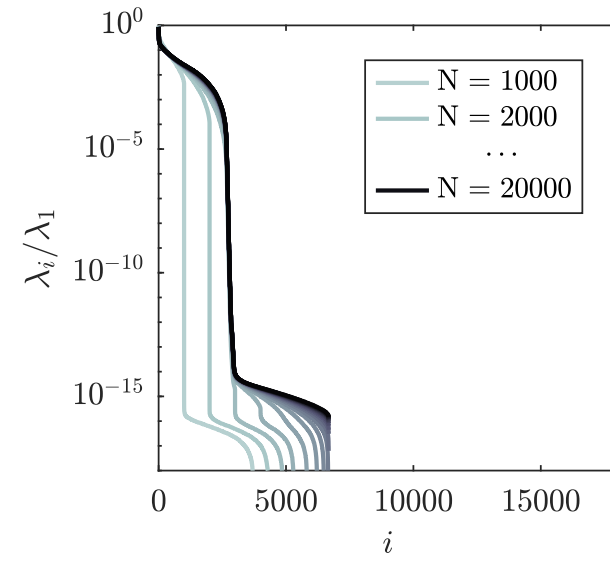

(a) $\mathrm{G}_{130}$

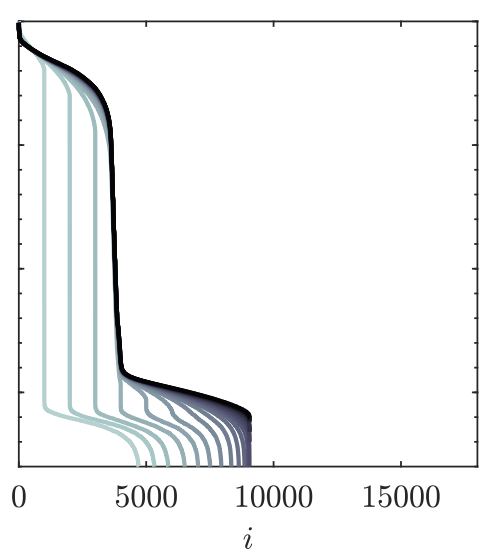

(b) $\mathrm{G}_{180}$

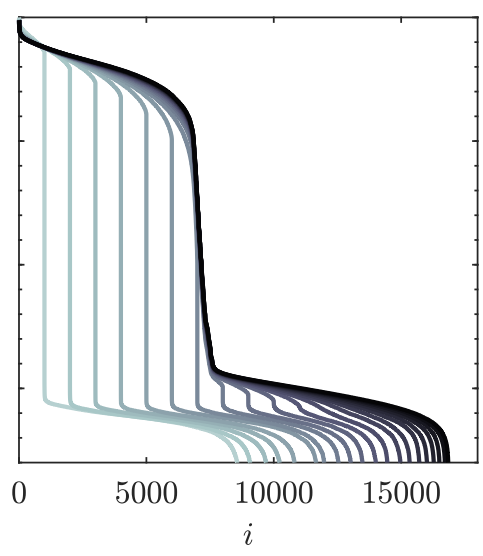

(c) $\mathrm{G}_{360}$

Fig. 10. (a) - (c) Spectrum (eigenvalues $\left.\lambda_{i}\right)$ of the covariance matrix $\Gamma_{\varepsilon}$ with varying number of samples $(N=1000,2000, \cdots, 20000)$ for sensor geometries $\mathrm{G}_{130}-\mathrm{G}_{360}$ with uncertainty $\mathrm{P}_{\mathrm{ANG}, 3}$. The eigenvalues have been scaled by $\lambda_{1}^{-1}$.

initial pressure lied within the credible interval in all of the posterior densities computed using the approximation error approach.

In addition to the simulation studies, the approximation error approach was evaluated using experimental data. Compared to the simulation study, modeling of the experimental setup introduces additional possible sources of modeling errors such as uncertainties in the speed of sound. Furthermore, the errors due to uncertainties in ultrasound sensor locations vary greatly depending on the characteristics of the ultrasound sensor and measurement geometry. Such characteristics include frequency response, size, directivity, position of the ultrasound sensor, and number of sensors or measurement angles. That is, approximation errors are system-specific.

In this work, sources of additional modeling errors due to sensor shape were minimized by using a quasi-3D model. This allowed for accurate modeling of the concave ultrasound sensors in three dimensions while producing computationally efficient 2D images. Furthermore, the temporal and spatial discretization were chosen such that the maximum frequency of $\sim 2 \mathrm{MHz}$ of the experimental data was well supported by both the spatial and temporal grids. Even though the large computational burden of 3D photoacoustic tomography was circumvented using the quasi-3D model, assumptions for the $\mathrm{z}$-directional homogeneity cannot be made in a general situation. Furthermore, when using denser spatial and temporal discretization in three dimensions, computation and storage of the discrete operator $K$ may not be feasible. In this case, iterative approaches utilizing matrix-free modeling could be utilized [13], [42].

In the results of the experimental studies, the imaged target was clearly visible in the reconstructions using the accurate sensor locations even though the image quality was hindered by the relatively low signal to noise ratio due to LED-illumination. The results using the inaccurate sensor locations and the enhanced error model were comparable to the simulation study. That is, modeling errors due to the radial uncertainties resulted in significant artifacts in the reconstructed images. Furthermore, artifacts were significantly reduced using the Bayesian error modeling.

Results obtained by utilizing modeling of errors show great promise in compensating uncertainties in ultrasound sensor locations. In accordance with these results, this method could be utilized in situations where the measurement of the exact sensor locations is challenging. This type of measurement setups include hand-held sensors, setups where the sensors are manually placed on a boundary of the target, or setups where movement of the sensors is a problem.

\section{APPENDIX}

\section{IMPACT OF THE NUMBER OF SAMPLES $N$ USED IN} COMPUTATION OF THE APPROXIMATION ERROR STATISTICS

The amount of samples $N$ used in computing the approximation error statistics is an important factor when considering the performance of the approximation error method. An insufficient amount of samples may result in a situation where the covariance of the approximation error does not reflect the error statistics accurately enough. Computing too many samples can, on the other hand, result in an unnecessarily heavy computational burden. A suitable value for $N$ can be estimated, for example, by inspecting the spectrum of the approximation error covariance matrix $\Gamma_{\varepsilon}$. As $N$ increases, the spectrum will eventually stagnate and only minor changes are seen with increasing $N$.

Illustrations of spectra for $\Gamma_{\varepsilon}$ of the simulation studies with sensor geometries $G_{130}-G_{360}$, uncertainty $P_{A N G, 3}$, and varying number of samples $N$ are shown in Fig. 10. It can be seen that the spectra stagnate at $N$ which is dependent on the number of sensors. Furthermore, a sample count of $N=10000$ would be sufficient in the cases of sensor geometries $\mathrm{G}_{130}$ and $\mathrm{G}_{180}$, whereas $N=20000$ samples are required in the case of the full view geometry $G_{360}$. This is due to the fact, that the size and complexity of $\Gamma_{\varepsilon}$ increases with increasing number of sensors. Sample counts of $N=20000$ (simulation study) and $N=10000$ (experimental study) used in this work were chosen based on this observation. 


\section{REFERENCES}

[1] P. Beard, "Biomedical photoacoustic imaging," Interface Focus, vol. 1, no. 4, pp. 602-631, Aug. 2011.

[2] C. Li and L. V. Wang, "Photoacoustic tomography and sensing in biomedicine," Phys. Med. Biol., vol. 54, no. 19, pp. R59-R97, Sep. 2009.

[3] L. A. Kunyansky, "A series solution and a fast algorithm for the inversion of the spherical mean Radon transform," Inverse. Probl., vol. 23, no. 6, pp. 11-20, Nov. 2007.

[4] S. J. Norton and M. Linzer, "Ultrasonic reflectivity imaging in three dimensions: Exact inverse scattering solution for plane, cylindrical and spherical apertures," IEEE. Trans. Med. Imag., vol. 28, no. 2, pp. 202 220, Feb. 1981.

[5] M. Xu and L. V. Wang, "Universal back-projection algorithm for photoacoustic computed tomography," Phys. Rev. E., vol. 71, no. 1, p. 016706, Jan. 2005.

[6] L. A. Kunyansky, "Explicit inversion formulae for the spherical mean Radon transform," Inverse. Probl., vol. 23, no. 1, pp. 373-383, Jan. 2007.

[7] Y. Hristova, P. Kuchment, and L. Nguyen, "Reconstruction and time reversal in thermoacoustic tomography in acoustically homogeneous and inhomogeneous media," Inverse. Probl., vol. 24, no. 5, p. 055006, Aug. 2008.

[8] P. Burgholzer, G. J. Matt, M. Haltmeier, and G. Paltauf, "Exact and approximative imaging methods for photoacoustic tomography using an arbitrary detection surface," Phys. Rev. E., vol. 75, no. 4, p. 046706, Apr. 2007.

[9] X. L. Deán-Ben, A. Buehler, V. Ntziachristos, and D. Razansky, "Accurate model-based reconstruction algorithm for three-dimensional optoacoustic tomography," IEEE. Trans. Med. Imag., vol. 31, no. 10, pp. 1922-1928, Oct. 2012.

[10] S. R. Arridge, P. Beard, M. M. Betcke, B. T. Cox, N. Huynh, F. Lucka et al., "Accelerated high-resolution photoacoustic tomography via compressed sensing," Phys. Med. Biol., vol. 61, no. 24, pp. 8908-8940, Dec. 2016.

[11] J. Tick, A. Pulkkinen, and T. Tarvainen, "Image reconstruction with uncertainty quantification in photoacoustic tomography," J. Acoust. Soc. Am., vol. 139, no. 4, pp. 1951-1961, Apr. 2016.

[12] M. Sun, N. Feng, Y. Shen, J. Li, L. Ma, and Z. Wu, "Photoacoustic image reconstruction based on Bayesian compressive sensing algorithm," Chinese. Opt. Let., vol. 9, no. 6, p. 061002, Jun. 2011.

[13] J. Tick, A. Pulkkinen, F. Lucka, R. Ellwood, B. T. Cox, J. P. Kaipio et al., "Three dimensional photoacoustic tomography in Bayesian framework," J. Acoust. Soc. Am., vol. 144, no. 4, pp. 2061-2071, Sep. 2018

[14] J. P. Kaipio and E. Somersalo, Statistical and Computational Inverse Problems. New York, NY, USA: Springer, 2005.

[15] A. Tarantola, Inverse Problem Theory and Methods for Model Parameter Estimation. Philadelphia, PHL, USA: SIAM Society for Industrial and Applied Mathematics, 2005.

[16] X. Jin and L. V. Wang, "Thermoacoustic tomography with correction for acoustic speed variations," Phys. Med. Biol., vol. 51, no. 24, pp. 6437-6448, Nov. 2006.

[17] T. P. Matthews, J. Poudel, L. Li, L. V. Wang, and M. A. Anastasio, "Parametrized joint reconstruction of the initial pressure and sound speed distributions for photoacoustic computed tomography," Soc. Ind. Appl. Math., vol. 11, no. 2, pp. 1560-1588, Jun. 2018

[18] J. Tick, A. Pulkkinen, and T. Tarvainen, "Modelling of errors due to speed of sound variations in photoacoustic tomography using a Bayesian framework," Biomed. Phys. Eng. Express, vol. 6, no. 1, p. 015003, Nov. 2019.

[19] K. Wang, R. Su, A. A. Oraevsky, and M. A. Anastasio, "Investigation of iterative image reconstruction in three-dimensional optoacoustic tomography," Phys. Med. Biol., vol. 57, no. 17, pp. 5399-5423, Dec. 2012.

[20] K. Mitsuhashi, K. Wang, and M. A. Anastasio, "Investigation of the farfield approximation for modeling a transducer's spatial impulse response in photoacoustic computed tomography," Photoacoustics, vol. 2, no. 1, pp. 21-32, Mar. 2014.

[21] T. Tarvainen, A. Pulkkinen, B. T. Cox, J. P. Kaipio, and S. R. Arridge, "Bayesian image reconstruction in quantitative photoacoustic tomography," IEEE Trans. Med. Imag., vol. 32, no. 12, pp. 2287-2298, Aug. 2013.

[22] A. Pulkkinen, V. Kolehmainen, J. P. Kaipio, B. T. Cox, S. R. Arridge, and T. Tarvainen, "Approximate marginalization of unknown scattering in quantitative photoacoustic tomography," Inv. Probl. Imag., vol. 8, no. 3, pp. 811-829, Aug. 2014.
[23] N. Hänninen, A. Pulkkinen, and T. Tarvainen, "Image reconstruction with reliability assessment in quantitative photoacoustic tomography," J. Imaging., vol. 4, no. 148, Dec. 2018.

[24] S. R. Arridge, J. P. Kaipio, V. Kolehmainen, M. Schweiger, E. Somersalo, T. Tarvainen et al., "Approximation errors and model reduction with an application in optical diffusion tomography," Inverse. Probl., vol. 22, no. 1, pp. 175-195, Jan. 2006.

[25] T. Tarvainen, V. Kolehmainen, A. Pulkkinen, M. Vauhkonen, M. Schweiger, S. R. Arridge et al., "An approximation error approach for compensating for modelling errors between the radiative transfer equation and the diffusion approximation in diffuse optical tomography," Inverse. Probl., vol. 26, no. 1, p. 015005, Dec. 2009.

[26] V. Kolehmainen, M. Schweiger, I. Nissilä, T. Tarvainen, S. R. Arridge, and J. P. Kaipio, "Approximation errors and model reduction in threedimensional diffuse optical tomography," J. Opt. Soc. Am. A., vol. 26, no. 10 , pp. 2257-2268, Oct. 2009.

[27] J. Koponen, T. Huttunen, T. Tarvainen, and J. P. Kaipio, "Bayesian approximation error approach in full-wave ultrasound tomography," IEEE Trans. Ultrason., Ferroelectr., Freq. Control, vol. 61, no. 10, pp. 1627-1637, Sep. 2014

[28] M. Mozumder, T. Tarvainen, S. R. Arridge, J. P. Kaipio, and V. Kolehmainen, "Compensation of optode sensitivity and position errors in diffuse optical tomography using the approximation error approach," J. Biomed. Opt., vol. 4, no. 10, pp. 2015-2031, Sep. 2013.

[29] M. Mozumder, T. Tarvainen, J. P. Kaipio, S. R. Arridge, and V. Kolehmainen, "Compensation of modeling errors due to unknown domain boundary in diffuse optical tomography," J. Opt. Soc. Am. A., vol. 31 , no. 8 , pp. 1847-1855, Aug. 2014

[30] J. Heino, E. Somersalo, and J. P. Kaipio, "Compensation for geometric mismodelling by anisotropies in optical tomography," Opt. Exp., vol. 13, no. 1, pp. 296-308, Jan. 2005.

[31] J. Heiskala, V. Kolehmainen, T. Tarvainen, J. P. Kaipio, and S. R. Arridge, "Approximation error method can reduce artifacts due to scalp blood flow in optical brain activation imaging," J. Biomed. Opt., vol. 17, no. 9, p. 096012, Sep. 2012.

[32] T. Lähivaara, N. D. Ward, T. Huttunen, J. Koponen, and J. P. Kaipio, "Estimation of aquifer dimensions frompassive seismic signals with approximatewave propagation models," Inverse. Probl., vol. 30, p. 015003 , Dec. 2014.

[33] T. Lähivaara, N. D. Ward, T. Huttunen, Z. Rawlinson, and J. P. Kaipio, "Estimation of aquifer dimensions from passive seismic signals in the presence of material and source uncertainties," Geophys. J. Int., vol. 200, no. 3, pp. 1662-1675, Mar. 2015.

[34] A. D. Pierce, Acoustics - An introduction to physical principles and its applications. Melville, Ny, USA: Acoust. Soc. Am., 1981.

[35] B. E. Treeby and B. T. Cox, "k-Wave: MATLAB toolbox for the simulation and reconstruction of photoacoustic wave fields," J. Biomed. Opt., vol. 15, no. 2, p. 021314, Mar 2010.

[36] J. P. Kaipio and E. Somersalo, "Statistical inverse problems: Discretization, model reduction and inverse crimes," J. Comput. Appl. Math., vol. 198, no. 2, pp. 493-504, Jan. 2007.

[37] V. Kolehmainen, T. Tarvainen, S. R. Arridge, and J. P. Kaipio, "Marginalization of uninteresting distributed parameters in inverse problems - application to diffuse optical tomography," Int. J. Uncertainty. Quant., vol. 1, no. 1, pp. 1-17, Jan. 2011.

[38] A. Nissinen, L. M. Heikkinen, and J. P. Kaipio, "The Bayesian approximation error approach for electrical impedance tomography experimental results," Meas. Sci. Technol., vol. 19, no. 1, p. 015501, Nov. 2008.

[39] C. E. Rasmussen and C. K. I. Williams, Gaussian Processes for Machine Learning. Cambridge, MA, USA: MIT Press, 2006.

[40] J. Leskinen, A. Pulkkinen, J. Tick, and T. Tarvainen, "Photoacoustic tomography setup using LED illumination," in Opto-Acoustic Methods and Applications in Biophotonics IV, V. Ntziachristos and R. Zemp, Eds., vol. 11077, Jul. 2019, pp. $57-68$

[41] Y. Xu, L. V. Wang, G. Ambartsoumian, and P. Kuchment, "Reconstructions in limited-view thermoacoustic tomography," Med. Phys., vol. 31, no. 4, pp. 724-733, Apr. 2004.

[42] S. R. Arridge, M. M. Betcke, B. T. Cox, F. Lucka, and B. E. Treeby, "On the adjoint operator in photoacoustic tomography," Inverse. Probl., vol. 32, no. 22, p. 115012, Oct. 2016. 